\title{
Investigation of moving striations in the low pressure krypton-mercury vapor discharges
}

\author{
Toshiro KAJIWARA* and Yoshinori ANZAI*
}

\begin{abstract}
When the fluorescent lamp, which is filled low pressure krypton gas and saturated mercury vapor, is operated on a commercial ballast working at 50 or $60 \mathrm{~Hz}$, it has the characteristics as follows; as the electric power of the lamp is reduced at room temperature, it can sustain the same light emission characteristics as the general one. But when the ambient temperature around the lamp is lower than the room temperature, the fluctuation of light emission appears below the critical temperature and the light emission is reduced remarkably.

To investigate these defective phenomena in the lamp, we studied the properties of the discharge voltage-current, the fluctuation frequencies and wavelength of moving lights which depend on the ambient temperature at the constant pressure of the filling gas. As the results, we obtained conclusions as follows.

(1) The fluctuation of light emission behaves like as the positive moving striations.

(2) The temperature at which moving striations appear is inferred to be determined by a mole fraction and a mole number in the discharge tube of krypton-mecury vapor mixture.

(3) These moving striations have similar characteristics to "r" wave which is based on the fluctuation of ion density in Ne gas discharge.

(4) In this temperature region in which moving striations appear, the excitation of krypton gas is activated, and the light emission of mercury line decreases.
\end{abstract}

\section{Introduction}

A fluorescent lamp filled with low pressure krypton gas and saturated mercury vapor operated on the commercial frequencies has the superior characteristics to argon-filled one from the point of view of energy saving. But the lamp has the defects of the unstable discharges, the fluctuation of light emission and the decrease of luminous flux as the ambient temperature is decreased below the special temperature.

In 1977 , G. S. Evans reported that these phenomena depended on moving striations. ${ }^{1)}$ But these phenomena have not sufficiently been explained in his paper. Ever since, the intensive studies have not been reported on moving striations which occur in the krypton-filled fluorescent lamp operated below

\footnotetext{
"Mitsubishi Electric Corp., Consumer Products Research Lab. A part of this paper has been published as a preprint of Illum. Engng. Inst. Japan Conference at Tokyo in $\mathbf{1 9 8 1 .}$
}

the special ambient temperature at the commercial frequencies.

The first study of moving striations was reported by F.W. Aston and T. Kikuchi. ${ }^{2)}$ In Japan, H. Yoshimoto indicated that the positive moving striations $^{* *}$ in DC glow discharges are caused by the fluctuation of electron density and based on waves of the interaction between electron density toward the anode and the space charge toward the cathode. ${ }^{3) 4}$ In order to clarify the wave nature of moving striations, the dispersion relation was measured in rare gases, rare gas-mercury vapor systems, and hydrogen gas by modulating the DC discharge current at an audiofrequency signals. As the results, it was found that the positive moving striations are approximated by a hyperbolic curve and the negative moving striations*** by a straight

** The waves of which the phase velosity propagates to the cathode and the group velosity to the anode were defined by $T$. Donauhe. $\left.{ }^{5}\right)$

*** The waves of which the phase and group velocity propagate to the anode. 
line in $\omega-k$ frame ${ }^{*}$, and the positive moving striations are classified into "rare gas type" and "rare gas-mercury type" and the negative moving striations are found in molecular gases. ${ }^{6)}$

In the next place, we summarize the results of literatures of moving striations in foreign countries. L. Pekareki) theoretically investigated the characteristics of moving striations by modulating the DC discharge current. A. Garscadden ${ }^{3)}$ considered the Pekarek theory in relation to the theory of H.S. Robertson ${ }^{9}$ who reflected the possible important role of metastable atoms and discussed the instabilities of moving striations. K. Wojaczeck ${ }^{10)}$ took account of heat transfer in the discharge plasmas, and K.W. Gentle ${ }^{1 x}$ ) discussed the ionization waves which were based on the Boltzmann equation. Since M. Novak ${ }^{12}$ ) discovered three kinds of self-excited waves in the discharge current below the Pupp's critical current ${ }^{13)}$ (1960), P. Pekarek and his co-workersi) (1970) compared his theoretical results with the experimental ones obtained from a wave packet excited by applying a pulsed voltage and concluded that the $r$ wave is induced by the fluctuation of ion density, the $p$ wave is caused by the fluctuation of metastable atom according to the analysis of the hydrodynamic equations including metastable atoms, and the $s$ wave is due to the space resonance of the $p$ wave by solving the Boltzmann equations for electrons.

The researches of moving striations have declined, but they have progressed as the precise measurements and the theoretical analysis of moving striations have been performed for the purpose of analysing the low frequency noise of gas laser. Though the previous studies have only explained the moving striations in DC discharges, the experimental and theoretical analysis of moving striations which occur in $\mathrm{AC}$ discharge at the practical low frequencies like in the practical fluorescent lamps still have never been reported.

In this paper, we discussed about the cause and the behaviours of the fluctuation of light waves*: which occur in the fluorescent lamp operated on the commercial ballast at AC below the special temperature from the point of view of the experimental analysis and results of previous literatures.

\section{Samples}

The discharge tubes, which were used for the experiments, exhausted by high vacuum pump (below $1.0 \times 10^{-4}$ Torr $^{2}$ ) were filled with krypton gas ( 1 to 5 Torr) and excess drops of mercury.

The discharge distance between electrodes was $1,100 \mathrm{~mm}$ and the inner diameter was $30 \mathrm{~mm}$. The electrodes used were for a general $40 \mathrm{~W}$ fiuorescent lamp.

In order to observe moving waves with high

* $\omega-k$ frame is composed of a wave number $k$ and an angular frequency $\omega$.

$*$ In this report, we call the fluctuation of luminous flux the light waves. speed, we used the clear discharge tubes without a lumminescent inside coating for all experiments.

\section{Experimental equipments and procedures}

\section{1 The observation of light waves by using a general and high speed camera}

The direction, the velocity and the wavelength of light waves were continuously observed by using a $16 \mathrm{~mm}$ high speed camera (HYCAM) in a constant temperature room. This room was kept 10 to $20^{\circ} \mathrm{C}$ with the accuracy $\pm 0.5^{\circ} \mathrm{C}$. And we used a conventional camera for observing an outline of moving striations (shutter speed: $1 / 60 \mathrm{sec}$.).

The dependency of light waves on the ambient temperature was observed at two points near the center and electrode of discharge tube. The high speed camera was adjusted from 800 to 2000 (frames/sec.).

\subsection{The measurements of light wave by using the electrical equipment}

To investigate the characteristics of light waves and the electric properties depending on the ambient temperature, the experimental arrangement illustrated in Fig. 1 was prepared.

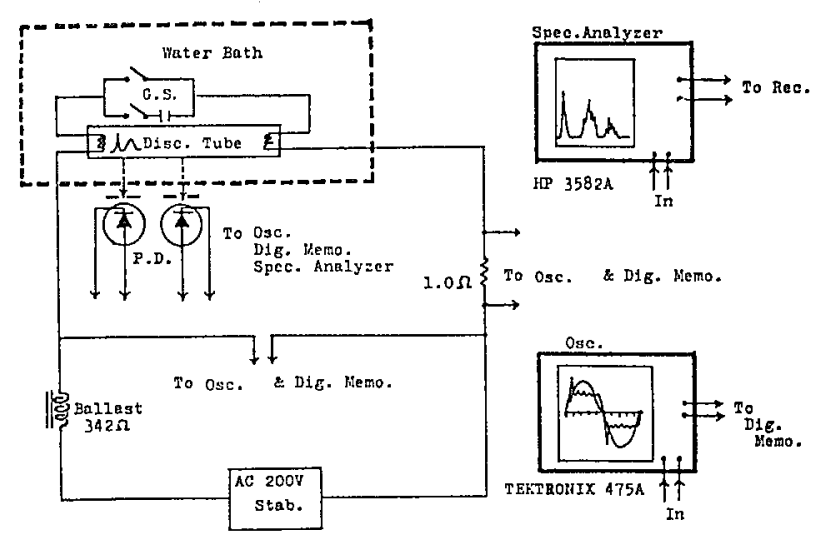

Fig. 1 Experimental arrangement

AC electric power supply was held constant within $\pm 0.1 \mathrm{~V}$ accuracy for lamp circuit by utilizing a stabilizer $(50 \mathrm{~Hz}, 200 \mathrm{~V})$.

A lamp ballast used was a reference ballast (impeadance: $342 \Omega$ at $20^{\circ} \mathrm{C}$ ).

$\mathrm{PD}$ indicates a silicon pin photodiode made by Hamamatsu TV (S1337-16BQ). This PD with thin plane has a good resolution to light wave, form, moreover, the aperture facing to thin $\mathrm{PD}$ was limited by a slit. The output of PD was amplified by Operation Amplifier (LH0032CG: N.S. Corp.).

A Spectrum Analyzer (HP3852A) was used for frequency analysis of light waves, and the spectrum was the average of 256 samplings. Fig. 2 (a) is an example of measurement in $\mathrm{Kr}-\mathrm{Hg}$ system ( $\mathrm{Kr}: 3$ Torr) at $15^{\circ} \mathrm{C}$ (in water bath), and Fig. 2 (b) is a 

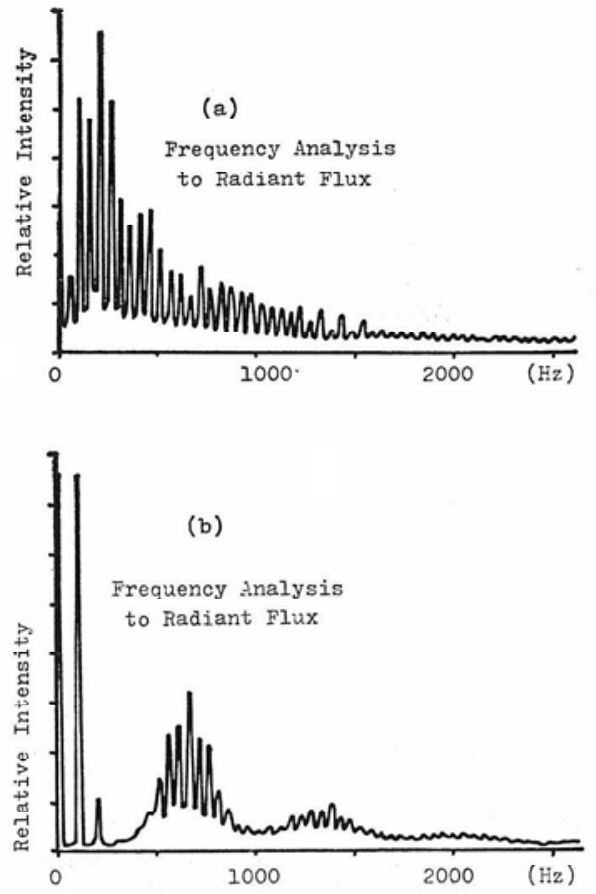

Fig. 2 Frequency analysis of light waves; (a) in $\mathrm{Kr}-\mathrm{Hg}$ system (Kr: 3.0 Torr), at $15^{\circ} \mathrm{C}$ in water bath, (b) in $\mathrm{Ar}-\mathrm{Hg}$ system (Ar: 2.65 Torr), at $5^{\circ} \mathrm{C}$ in water bath.

result in $\mathrm{Ar}-\mathrm{Hg}$ system (Ar: 2.65 Torr) to compare with $\mathrm{Kr}-\mathrm{Hg}$ system at $5^{\circ} \mathrm{C}$ (in water bath).

The frequency components of the lamp voltage and light profiles were analyzed by Band Pass Filter (FV-625A: NF Cir. Des. Block Corp.).

All data of light waves, the discharge voltage and current were introduced to Digital Memory (DM-901: Iwasaki Tsushinki Corp.) and to $\underline{\text { Oscillo- }}$ scope (Textronix 475A).
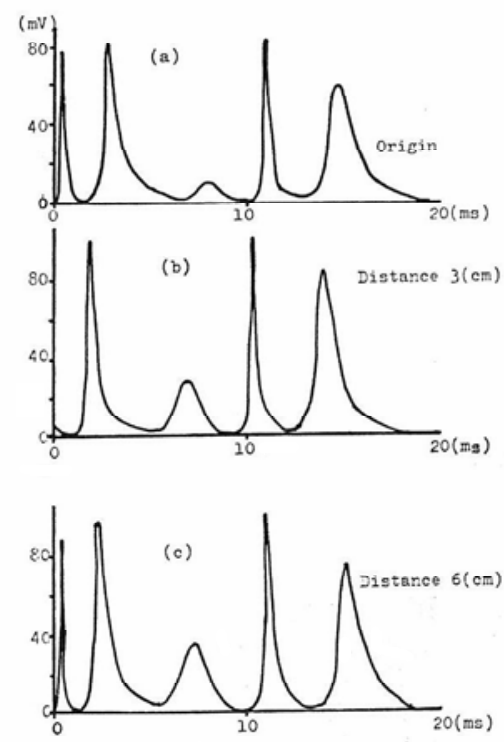

Fig. 3 Measurement of wavelength in $\mathrm{Kr}-\mathrm{Hg}$ system (Kr:5.0 Torr) at $5^{\circ} \mathrm{C}$ in water bath.
The direction of motion of light waves was determined by the phase deference between waveforms from two PDs. In this place, we explain the procedure of measurement with figures (Fig. 3). As one of PD was fixed (Fig. 3 (a)) and the other was moved along discharge tube (Figs. 3 (b) and (c)), the wave length of light waves was obtained as the distance of two PDs, at which the phases of light waveforms of two PDs have coincided.

Further, the ambient temperature of discharge tube was controled in water bath by utilizing the electronic thermoregurator with $\pm 0.1^{\circ} \mathrm{C}$ accuracy (CTE-220: Yamato Sci. Corp.).

\section{Experimental results}

\subsection{The direction of light waves}

Fig. 4 shows the photographs of light waves in $\mathrm{Kr}-\mathrm{Hg}$ system ( $\mathrm{Kr}: 5.0$ Torr) at $5.0^{\circ} \mathrm{C}$ (this temperature in air corresponding to $15^{\circ} \mathrm{C}$ in water bath). The photographs were shuttered by using a conventional camera at $1 / 60$ sec. Figs. 4 (a) and (b) show light waves near the electrode and the center of the discharge tube respectively.

To understand these light waves more precisely, we investigated the characteristics of light waves near the electrode and the center of the discharge tube by comparing with the frames of the photographs taken with high speed camera. As the results, we confirmed that the profiles of light waves were independent of the positions in the discharge tube.
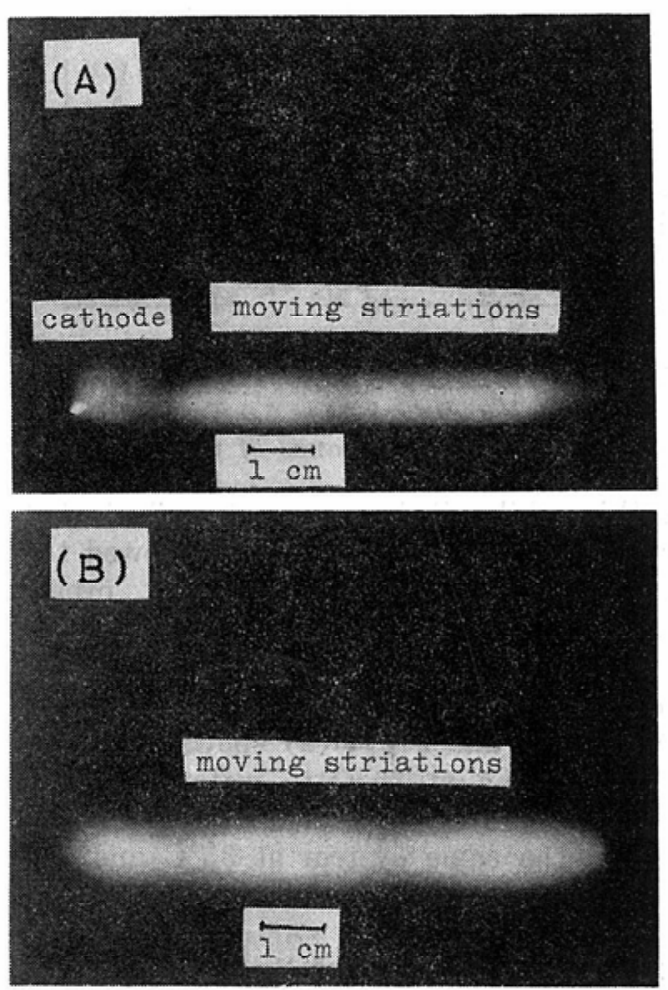

Fig. 4 Photographs of light waves in $\mathrm{Kr}$ $\mathrm{Hg}$ system (Kr: 5 Torr) at $5^{\circ} \mathrm{C}$ in air; (a) near the electrode (cathode) and $(b)$ near the center of the dischange tube. 
Fig. 5 shows the result of ellipsoidal light balls in $\mathrm{Kr}-\mathrm{Hg}$ system ( $\mathrm{Kr}: 0.5$ Torr) at $15^{\circ} \mathrm{C}$ (this temperature in air corresponding to $25^{\circ} \mathrm{C}$ in water bath), and it was found that these light waves moves to cathode. In Fig. 5, the arrows mean the directions of motion of the ellipsoidal light balls every millisecond.

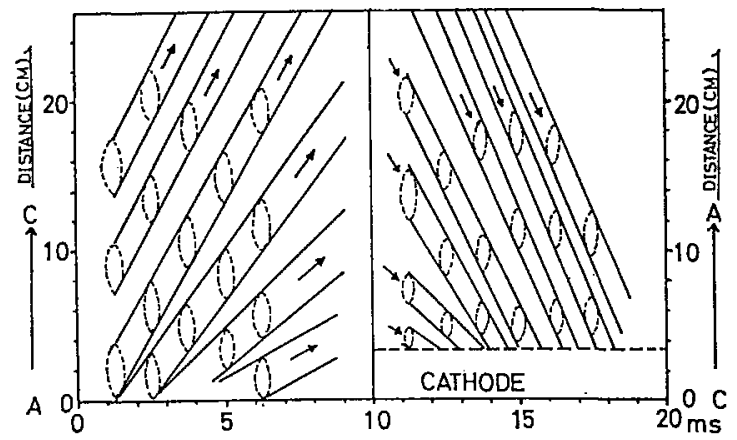

Fig. 5 Analysis of light waves by using a high speed camera in $\mathrm{Kr}$-Hg system ( $\mathrm{Kr}: 0.5$ Torr) at $15^{\circ} \mathrm{C}$ in air.

Fig. 6 illustrates light waves in $\mathrm{Kr}-\mathrm{Hg}$ system ( $\mathrm{Kr}$ : 5.0 Torr) at $25^{\circ} \mathrm{C}$ in water bath, and the arrows mean the directions of motion of light waves at the positions that the distance of two PDs is $3 \mathrm{~cm}$. These directions agreed with the results of the photographs by a high speed camera.

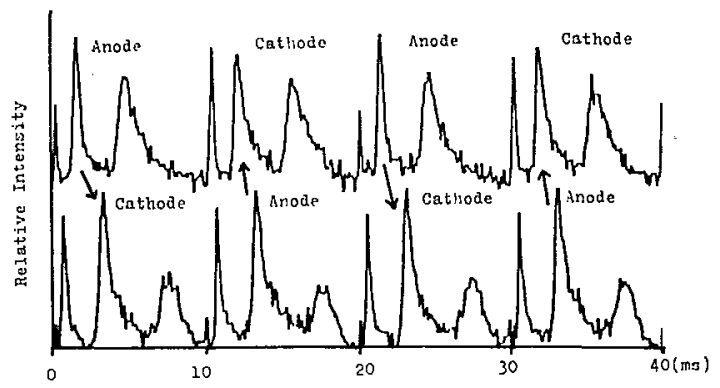

Fig 6 Directions of motions of light waves; $K r-H g$ system (Kr: 5.0 Torr), $25^{\circ} \mathrm{C}$ in water bath, distance of two PDs $3 \mathrm{~cm}$.

All this while, it has been described that the luminous flux was unstable, so we showed the discharge voltage-current and the light profiles in Fig. 7 and in Fig. 8, in order to compare the stable one. Fig. 8 (a) illustrates the discharge voltage and current profiles in $\mathrm{Kr}-\mathrm{Hg}$ system ( $\mathrm{Kr}: 3.0$ Torr) at $15^{\circ} \mathrm{C}$, and Fig. 7 (a) shows the profiles in the same system at $35^{\circ} \mathrm{C}$ in water bath. In the same way, Fig. 7 (b) and Fig. 8 (c) show the light profiles in the same system at $35^{\circ} \mathrm{C}$ and $15^{\circ} \mathrm{C}$ in water bath respectively.

\section{2 The velocity of light waves}

From Fig. 5, it was found that the wavelength was about $8 \mathrm{~cm}$ (the distance of the two ellipsoidal bright balls) and the velocity of light waves was about $35 \mathrm{~m} / \mathrm{sec}$. in $\mathrm{Kr}-\mathrm{Hg}$ system ( $\mathrm{Kr}$ : 0.5 Torr, $15^{\circ} \mathrm{C}$ in air). In the same way, it was confirmed
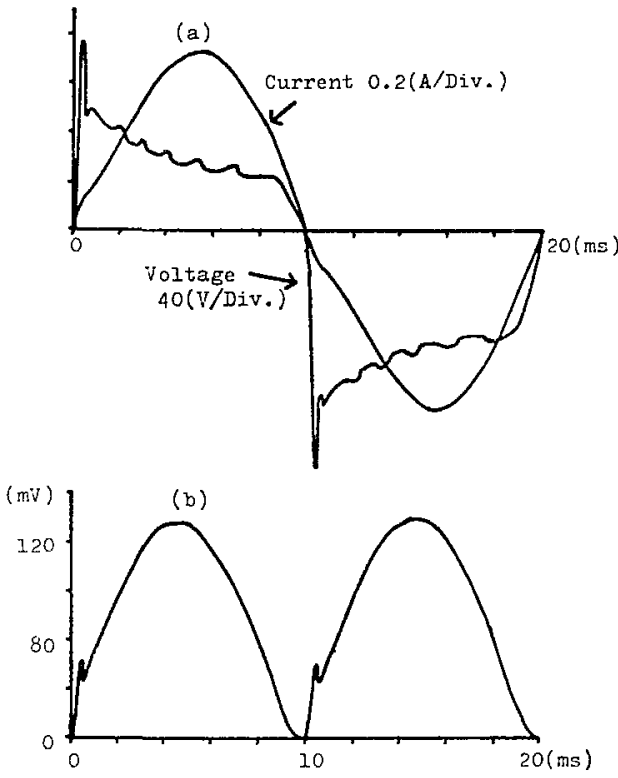

Fig 7 Wave profiles in $K r-H g$ system (Kr:3.0 Torr) at $35^{\circ} \mathrm{C}$ in water bath; (a) discharge voltage and current, and $(b)$ light waves.
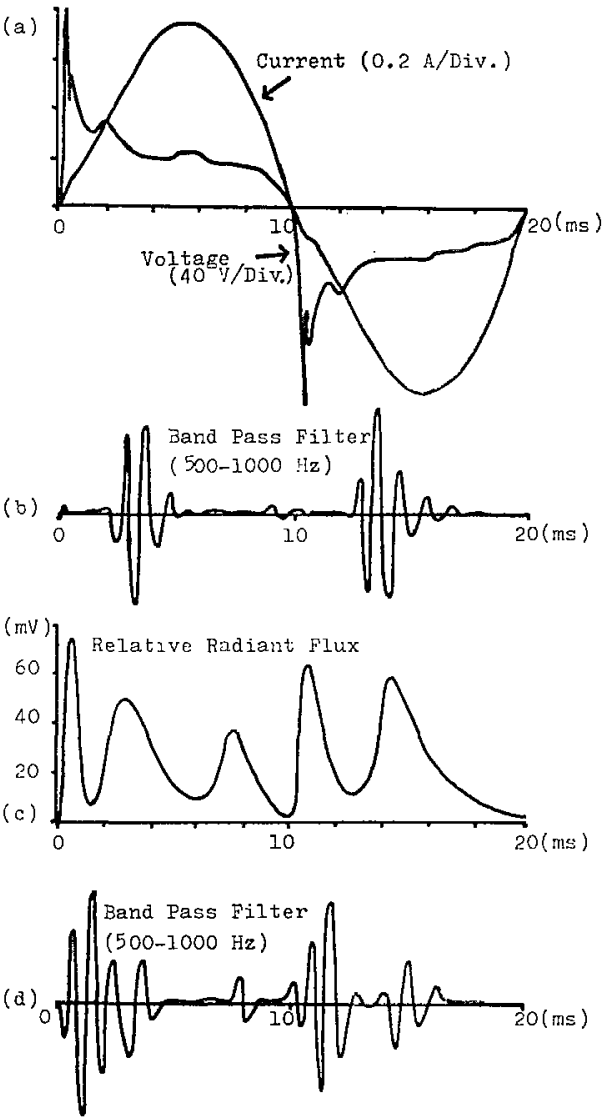

Fig. 8 Wave profiles in $\mathrm{Kr}$ - $\mathrm{Hg}$ system (Kr:3.0 Torr) at $15^{\circ} \mathrm{C}$ in water bath; (a) discharge voltage and current, (b) discharge voltage filtered through B.P. filter from 500 to $1000 \mathrm{~Hz}$, (c) light waves, and (d) light waves filtered through $B . P$. filter from 500 to $100 \mathrm{~Hz}$. 
that the wavelength was about $4 \mathrm{~cm}$ and the velocity was about $9 \mathrm{~m} / \mathrm{sec}$. in $\mathrm{Kr}-\mathrm{Hg}$ system ( $\mathrm{Kr}: 5.0$ Torr, $15^{\circ} \mathrm{C}$ in air). In these photographs by a high speed camera, it was observed that the velocity of light waves increased as the discharge current rose. In this place, Figs. 4 and 5 were illustrated the photographs in order to acquaint the behaviours of the ellipsoidal bright balls, but others by a high speed camera were too dark to show in this paper. And we were not able to show in Fig. 5, but it was observed that the radiant intensities show the maximum values near the reignition spikes and decrease as the discharge current increases from the results by a high speed camera.

The phase velocity of light waves was calculated by the product of the wavelength and frequency of light waves, obtained by PD measurement. As the results, the phase velocity and the wavelength of light waves were in the region of 8 to 45 (m/sec.) and 4 to $9(\mathrm{~cm})$ according to the experimental conditions done so far and were summarized in Table 1. These results agreed approximately with the values of the high speed camera.

Table 1 The dependency of the wave properties on the ambient temperature and filling gas pressure $(K r)$.

\begin{tabular}{|c|c|c|c|c|c|c|c|c|}
\hline & * & $5^{\circ} \mathrm{C}$ & $10^{\circ} \mathrm{C}$ & $15^{\circ} \mathrm{C}$ & $20^{\circ} \mathrm{C}$ & $25^{\circ} \mathrm{C}$ & $30^{\circ} \mathrm{C}$ & $35^{\circ} \mathrm{C}$ \\
\hline \multirow{3}{*}{1 Torr } & $\mathrm{f}$ & 200 & 250 & 280 & 380 & 550 & 690 & 900 \\
\hline & $\lambda$ & 9.2 & 8.8 & 8.6 & 7.8 & 7.0 & 5.3 & 5.0 \\
\hline & c & 18.4 & 22.0 & 24.0 & 29.6 & 38.5 & 36.6 & 45.0 \\
\hline \multirow{3}{*}{2 Torr } & $\mathrm{f}$ & - & 200 & 200 & 250 & 350 & 520 & - \\
\hline & $\lambda$ & - & 7.5 & 7.3 & 7.0 & 6.0 & 4.7 & - \\
\hline & c & - & 15.0 & 14.6 & 17.5 & 21.0 & 24.4 & - \\
\hline \multirow{3}{*}{3 Torr } & $\mathrm{f}$ & 200 & 220 & 240 & 280 & 340 & 560 & - \\
\hline & $\lambda$ & 6.2 & 6.2 & 6.0 & 5.5 & 5.0 & 4.8 & - \\
\hline & c & 12.4 & 13.6 & 14.4 & 15.4 & 17.0 & 26.9 & - \\
\hline \multirow{3}{*}{4 Torr } & f & - & - & 200 & 210 & 250 & 420 & - \\
\hline & $\lambda$ & - & - & 6.1 & 6.1 & 5.5 & 4.4 & - \\
\hline & c & - & - & 12.2 & 12.8 & 13.8 & 18.5 & - \\
\hline \multirow{3}{*}{5 Torr } & $f$ & 200 & 200 & 200 & 200 & 200 & 240 & 500 \\
\hline & $\lambda$ & 4.0 & 4.0 & 4.0 & 4.0 & 4.0 & 4.0 & 3.8 \\
\hline & c & 8.0 & 8.0 & 8.0 & 8.0 & 8.0 & 9.6 & 19.0 \\
\hline
\end{tabular}

f: Optical Frequency $(\mathrm{Hz}), 2$ : Wavelength of Striations $(\mathrm{cm})$, C: Phase Velocity $(\mathrm{m} / \mathrm{sec}$.)

\section{3 The dependency of the properties of light waves on the ambient temperature}

As described above, the fluctuation phenomena of luminous flux, which occur with decrease of the ambient temperature of discharge tube, are different from the previous papers about moving striations originated in the Pupp's critical current.

The dependency of the wavelength and the frequency of light waves on the filling gas pressure $(\mathrm{Kr})$ and the ambient temperature are summarized in Table 1.

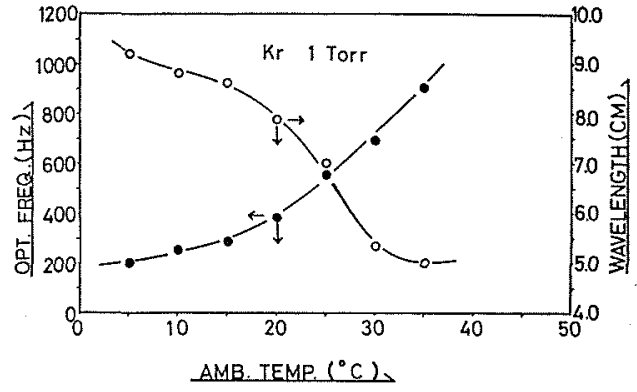

Fig. 9 The dependency of wavelength and frequency on the ambient temperature in $\mathrm{Kr}-\mathrm{Hg}$ system (Kr: 1.0 Torr).

Fig. 9 shows the dependence of the wavelength of light waves on the ambient temperature. As the results, it was found as follows.

(1) The wavelength gets shorter as the ambient temperature rises and the filling gas pressure $\left(\mathrm{Kr}^{\mathrm{r}}\right)$ increases.

(2) The frequency becomes higher as the ambient temperature rises and the filling gas pressure $(\mathrm{Kr})$ reduces.

(3) The phase velocity, which is calculated from the product of the wavelength and the frequency show the same tendency as the characteristics of the frequency.

These results are illustrated in Figs. 10, 11 and 12.

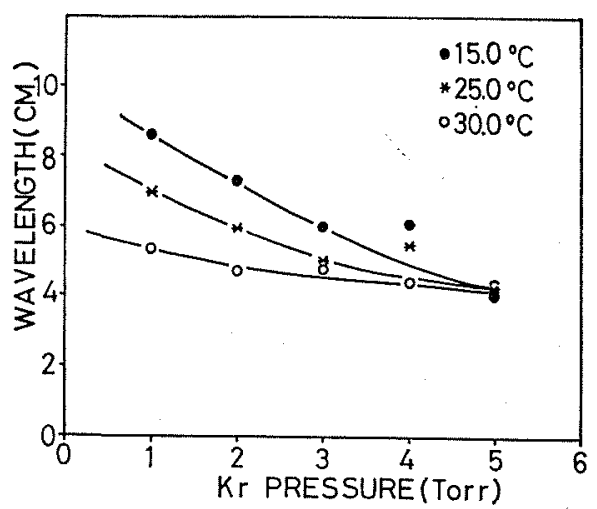

Fig. 10 The dependency of wavelength on filling gas pressure $(K r)$.

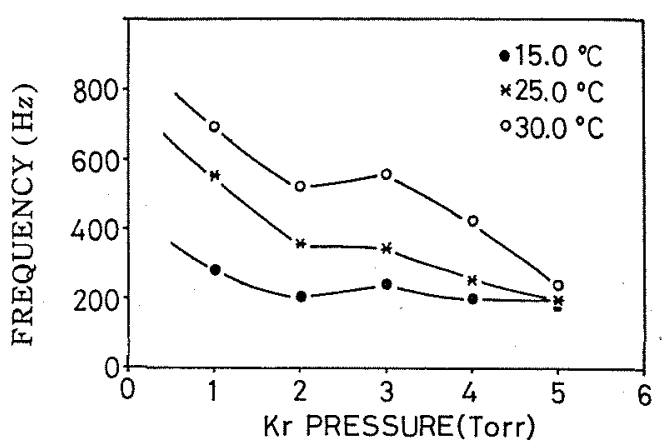

Fig. 11 The dependency of frequency on filling gas pressure $(K r)$. 


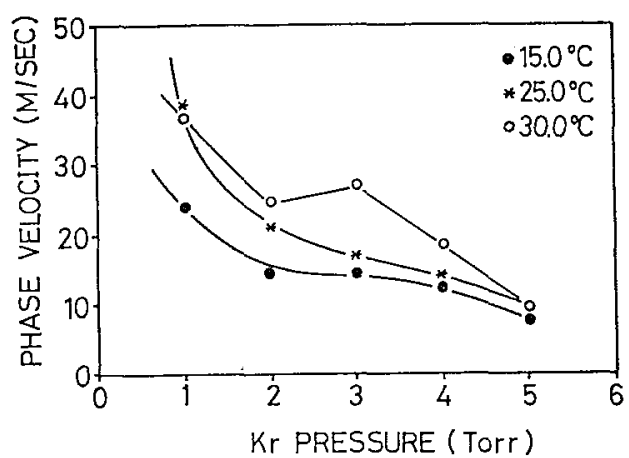

Fig. 12 The dependency of phase velocity on filling gas pressure $(K r)$.

\section{4 The dependency of the discharge voltage-current on the ambient temperature}

From the experimental results as described above, if the characteristics of light waves change suddenly as the ambient temperature increases, it can be expected that the properties of discharges simultaneously change too. Then, we examined the dependency of the discharge voltage and current on the ambient temperature in the same way as analysis of light waves. Fig. 13 is an example for their experiments.

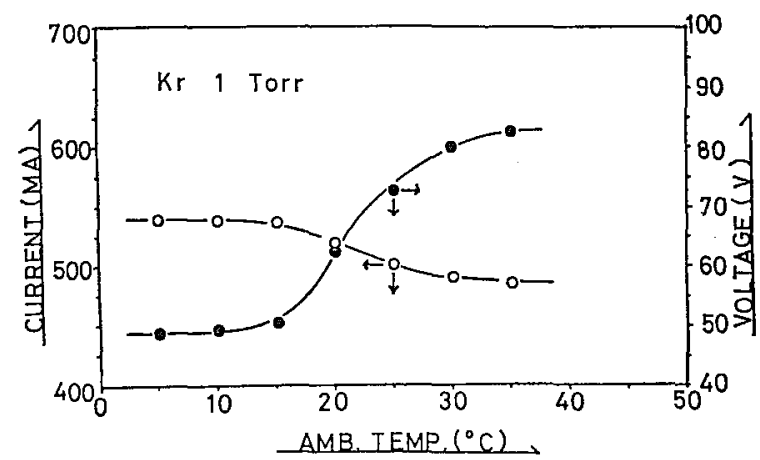

Fig. 13 The dependency of discharge voltage and current on the ambient temperature in $K r-H g$ system ( $K r: 1.0$ torr).

As the result, it was confirmed that the discharge voltage and current change suddenly at the same ambient temperature at which the wavelength of light waves change rapidly. The points of inflection to the ambient temperature which the wavelength, the discharge voltage and current have at the krypton gas pressure ( 1 to 5 Torr) were averaged. We defined them as the critical temperature at the each filling krypton gas presure. Fig. 14 shows the relation between the critical temperature and the pressure filled with krypton gas.

As described above, it was found that the critical temperature decreases as the pressure filled with krypton gas increases.

At last, we added the results of the discharge voltage and the light wave profiles by B.P. filter from 500 to $1,000 \mathrm{~Hz}$ in Figs. 8 (b) and (d) respectively.

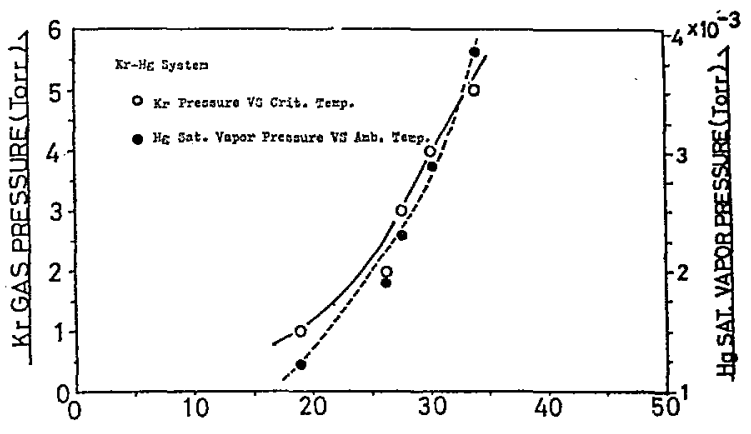

IEMP $\left({ }^{\circ} \mathrm{C}\right.$ )

Fig. 14 The dependency of the critical temperature on filling krypton gas pressure and the relation between $\mathrm{H} g$ saturated vapor pressure and the ambient temperature.

\section{Discussion}

\subsection{The relation between light waves and moving striations}

It was certified that light waves appeared in $\mathrm{Kr}-\mathrm{Hg}$ system below the special temperature propagates from the anode to the cathode. According to the previous papers, it is known that moving striations are composed of the positive moving striations of which the phase velocity propagates from the anode to the cathode and the group velocity moves from the cathode to the anode and the negative moving striations which both the phase and group velocity move from the cathode to the anode. The phase velocities obtained by these measurements are in good agreement with the value of the previous paper ${ }^{6 / 8)}$ investigated on the DC glow discharges in the rare gases and the rare gas-mercury systems. Specially, the resonance point reported by $\mathrm{H}$. Yoshimoto consists with the value in this paper. As described above, it was found that the fluctuation of luminous flux on the fluorescent lamp in the $\mathrm{Kr}-\mathrm{Hg}$ system at the critical temperature is caused by the positive moving striations. When the lamp is operated at $50 \mathrm{~Hz}$, the velocity of moving striations is accelerated as the discharge current increases, and the intensity of luminous flux becomes the maximum near the reignition spikes according to the measurements by a high speed camera.

In the same way, it was confirmed that the above mentioned phenomena are the positive moving striations with the periodic changes which decrease as the discharge current increases.

\section{2 The classification of moving striations on the fluorescent lamp}

As described above, moving striations which appear below the Pupp's critical current in DC glow discharges can be classified into the $r$ wave caused by the fluctuation of ion density, the $p$ wave by the fluctuation of the metastable atom density and the $s$ wave explained as the space resonance of the $p$ wave. ${ }^{7)}$ And we elucidated the fluctuation phenomena appearing below the critical temperature in 
$\mathrm{Kr}-\mathrm{Hg}$. system as the positive moving striations.

We tried to classify these moving striations according to the previous papers. In this case, we referred to the recent paper by $K$. Ohe and $S$. Takeda. ${ }^{15)}$ They reported on the DC glow discharges in Ne gas (the positive moving striations) at the low current region as follows;

(1) the $r$ wave has the properties that the frequency increases as the gas temperature rises and decreases as the filling gas pressure ( $\mathrm{Ne}$ ) increases,

(2) the $p$ wave has the reverse tendencies,

(3) the $s$ wave has the same properties as the $p$ wave behaves, and

(4) the $r$ wave is excited and the $p$ wave is attenuated with increasing the discharge current in the region of a few $\mathrm{mA}$ to $1 \mathrm{~A}$.

As mentioned above, the results of our measurements agreed with the behaviours of the $r$ wave in Ne gas discharge.

\section{3 The relation between the critical temperature and the occurrence of moving striations}

We defined the critical temperature of moving striations in the experimental results, and described that the critical temperature depends on the pressure filled with krypton gas in $\mathrm{Kr}-\mathrm{Hg}$ system (Fig. 14), that is, on a mole fraction of krypton gas or mercury vapor. In this section, we discussed a few points about the reasons.

A. Garscadden $\left.{ }^{8}\right)$ developed the Pekarek theory about the instabilities of moving striations. In his paper, A. Garscadden described moving striations composed of stable and unstable waves in DC glow discharges in the region above $100 \mathrm{~mA}$, and he indicated as follows; if the width of modulating pulse becomes sufficiently long, moving striations will spread throughout the discharge tube.

Pekarek theory supposed that the Debye length is vanishingly small in comparison with the striation wavelength, and derived the one-dimensional linearized continuity equation for ion density. In the equation, it was discussed that the fluctuation of the electron temperature, which has a reciprocal relaxation length $\left(\mathrm{cm}^{-1}\right)$, and the ion density influence on the ion production rate, that is, the stabilities of the short and long wavelength fluctuation.

From our studies, moving striations appearing in the fluorescent lamp below the critical temperature spread throughout the discharge tube, so we presumed that the pulse width (Fig. 8 (b)) which occurs on the commercial operating circuit promotes the long wavelength fluctuation and the ion density fluctuation throughout the discharge tube. The fluctuation caused by one pulse at $50 \mathrm{~Hz}$ discharge will be relaxed in the region of the moving striation wavelength. As this phenomena appear the same behaviours like the ion density fluctuation $^{8) 15)}$, we inferred to be able to replace the phenomena with the relation between the densitydensity correlation and the phase transitions near the critical point in the previous literature. ${ }^{16)}$ That is, it was implied that the density-density correlations between krypton and mercury ions control the moving striation wavelength corresponding to the relaxation wavelength and the phase velocity depending on the relaxation time. In particular, interested in the binary system between krypton and mercury ions, we will be able to treat it as same phenomena as the phase transitions in the binary critical mixtures (for example, cyclohaxaneaniline system). ${ }^{16)}$ So we can understand this striking feature that the properties of rare gas appear in the fluorescent lamp below the critical temperature. We are going to report on the another paper for these results, but we still have observed that the krypton line $(557.03 \mathrm{~nm})$ increases and mercury line $(435.8 \mathrm{~nm})$ decreases suddenly in K:$\mathrm{Hg}$ system below the critical temperature. We showed this results in Fig. 15 for reference. The radiant intensities of these lines were obtained by using interference filter (halfwidth $2 \mathrm{~nm}$ ). From this results, we were able to explain the phase transition depending on the excited atom densities in $\mathrm{AC}$ discharges near the critical temperature.

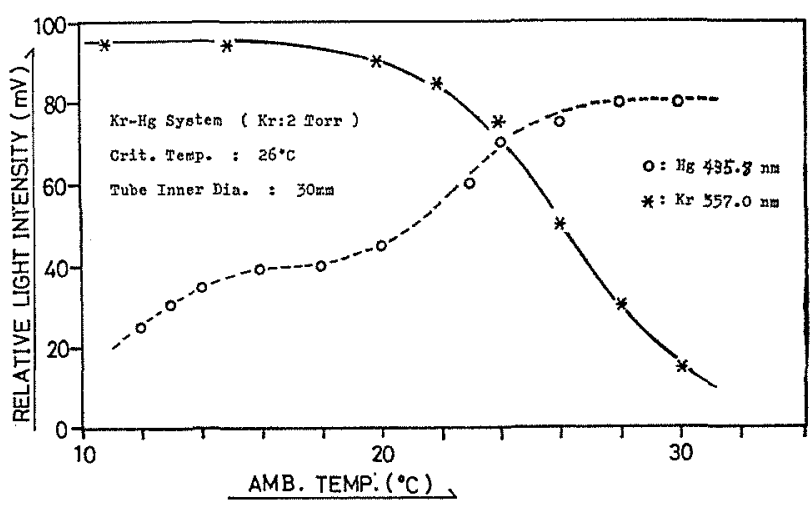

Fig. 15 The radiant intensities of krypton and mercury lines depending on the ambient temperature.

As considered above, we supposed moving striations caused in $\mathrm{Kr}-\mathrm{Hg}$ system at operating on the commercial ballast and frequencies below the critical temperature depend on the correlations between the ion densities ( $\mathrm{Kr}$ and $\mathrm{Hg}$ ions), and the critical temperature are defined by the number densities (mole number and mole fraction) between krypton and mercury atoms.

\section{Conclusions}

The phenomena such as the fluctuation of luminous flux occurring on the fluorescent lamp in $\mathrm{Kr}$ $\mathrm{Hg}$ system below the low temperature is concluded as follows.

(1) The fluctuation of light emission behaves like as the positive moving striations.

(2) The temperature at which moving striations appear is inferred to be determined by a mole fraction and a mole number (number density) in the discharge tube of krypton-mercury vapor mixture. 
(3) These moving striations have similar characteristics to " $r$ " wave which is based on the fluctuation of ion density in $\mathrm{Ne}$ gas discharges.

(4) In this temperature region in which moving striations appear, the excitation of krypton gas is activated, and the light emission of mercury line decreases.

\section{Acknowledgement}

We would like to particular thank Mr. M. Tsuchihashi of our laboratory for his valuable advice and encouragement throughout this work.

\section{References}

(1) Evans, G.S.: Light. Des. Appl. 4-7 (1974) 10.

(2) Aston, F.W. and Kikuchi, T.: Proc. Roy. Soc. 98 (1920) 50.

(3) Yoshimoto, H.: Nipponbutsurigakkaishi (in Japanese) 17-11 (1962) 790.

(4) Yoshimoto, H., Sato, M. and Nakao, Y.: J. Phys. Soc. Japan 13-7 (1958) 734 .
(5) Donauhe, T. and Dieke, G.H.: Phys, Rev. 81 (1951) 248

(6) Yoshimoto, H. and Yamashita, Y.: J. Phys. Soc. Japan 22-4 (1967) 1087.

(7) Pekarek, L. and Krejci, V.: Czech. J. Phys. B13 (1963) 881; ibid. B12 (1962) 296; ibid. B11 (1961) 729 Pekarek $L$. and et al.: ibid. B20 (1970) 879.

(8) Garscadden, A. and Bletzinger, P.: Phys. Fluids 12-9 (1969) 1833.

(9) Robertson, H.S.: Phys. Rev. 105 (1957) 368.

(10) Wojaczek, K.: Beitr. Plasmaphys. 2 (1962) 1.

(11) Gentle, K.W.: Phys. Fluids 9 (1966) 2203.

(12) Novak, M.: Czech. J. Phys. 10 (1960) 954.

(13) Pupp, W.: Phys. Z 33 (1932) 844.

(14) Michel, G., Drouet and Milos Sicha: Can. J. Phys. 46 (1968) 1787; ibid. 46 (1968) 1975; ibid. 46 (1968) 2491.

Michel, G. Drouet: ibid. 46 (1968) 2193.

(15) Ohe, K. and Takeda, S.: J. Phys. D 11 (1978) 2257.

(16) H. Eugene Stanley: "Introduction to Phase Transition and Critical Phenomena"; Claredon Press Oxford (1st. 1971).

Received 5 Nov. 1981; Revision Received 6 Jan. 1982 